\title{
A Comparison of Two Dietary Assessment Methods in a Mediterranean Lebanese Adults Population: A Food Frequency Questionnaire and a Dietary Recall
}

\author{
Maya Tueni ${ }^{1, *}$, Afifee Mounayar ${ }^{1}$, Ines Birlouez-Aragon ${ }^{2}$ \\ ${ }^{1}$ Department of Biology, Nutrition and Dietetics, Faculty of Sciences II, Lebanese University, Fanar, Lebanon \\ ${ }^{2}$ Spectralys Innovation Biocitech, 102 Gaston Roussel Street, 93230 Romainville, France
}

Copyright $(\mathrm{O} 2018$ by authors, all rights reserved. Authors agree that this article remains permanently open access under the terms of the Creative Commons Attribution License 4.0 International License

\begin{abstract}
Objective: To compare the results of a food frequency questionnaire (FFQ) of traditional Lebanese dishes with a weekly dietary recall (WDR). Methods: A food list of 56 traditional Lebanese dishes was constructed with color photographs to quantify portion sizes. 566 Lebanese, aged 20-62 years completed a FFQ before a WDR in the same day. Results: The FFQ overestimated the intakes of most food groups (10\%) and nutrients (7\%) in both sexes. Spearman correlation coefficients ranged from 0.22 for vegetables with meat to 0.95 for cereals, pastries and dairy products in men and from 0.30 for fish to 0.86 for dairy products in women (median: 0.53 for 10 food groups). $10 \%$ or less of participants were misclassified in opposite extreme quartiles (except for vegetables with meat in men $14.6 \%$ and fish in women $22.7 \%$ ). Spearman correlation coefficients ranged from 0.66 for retinol to 0.87 for folates in men and from 0.58 for PUFA to 0.69 for energy, fat, folates and potassium in women (median: 0.69 for 21 nutrients). $10 \%$ or less of participants were misclassified in opposite extreme quartiles. Conclusions: The FFQ of traditional food intake with a photographic atlas seems a reliable tool for dietary assessment in Mediterranean regions.
\end{abstract}

Keywords Food Frequency Questionnaire, Dietary Recall, Development and Evaluation, Dietary Assessment, Traditional Mediterranean Diet, Lebanon

\section{Introduction}

The traditional Mediterranean diet has a historical value, it is the most frequently investigated dietary pattern [1]. It is a typical diet followed by the people living in the countries surrounding the Mediterranean Sea. It is predominantly plant based, characterized by a high consumption of vegetables, fruits, non-refined cereals, legumes, nuts, seeds, olive oil as the principal source of fat, moderate amount of fish, poultry, eggs and dairy products, and low intake of red meat with a regular but moderate consumption of red wine. It is rich in dietary fiber, complex carbohydrate, vitamins, minerals, polyunsaturated fatty acids and phytochemicals [1-3]. It has been demonstrated to have a beneficial influence on health and an improvement in longevity. Particularly, greater adherence to the traditional Mediterranean diet has been inversely associated with chronic degenerative diseases, such as cardiovascular, neurodegenerative diseases, metabolic syndrome, obesity, cancers, liver disease, type 2 diabetes, depression, anxiety and overall total mortality [2-7].

Unfortunately the traditional Mediterranean diet has changed; it is progressively abandoned by the local populations in favor of a more westernized diet as clearly described in several studies $[1,7,8]$. Nevertheless, the traditional Mediterranean foods have left their imprints on contemporary dietary patterns. They are key elements for the dietary patterns in different countries and consequently are important to accurately estimate population dietary intakes $[1,8,9]$.

The Lebanese diet corresponds to the Mediterranean dietary pattern. However, the place of traditional meals in the common daily diet was not evaluated, and instruments that seek to assess traditional dietary patterns in Lebanon are missing.

There are several instruments and methods of dietary measurement available to epidemiological research: short-term dietary recall, dietary records, and food frequency questionnaires. Food frequency questionnaires (FFQ) and the repeated 24-hour recall are the most relevant tools [10-13]. FFQ are generally self-administrated but they can be administrated by an interviewer and participants are asked to report from a list of foods or foods groups how often and sometimes how much they ate over a specific period. The FFQ may 
include between 50 and 150 food items and frequency categories and/or types of food items may vary according to the objectives and designs of the study. FFQ aren't designed for estimating absolute nutrients intakes but they help to rank individuals by intake of selected foods or nutrients [10-13].

FFQ are popular for use in nutrition research because they are less burdensome than other dietary assessment methods [10-13]. They are widely used for dietary assessments in epidemiologic studies [10-13] due to their cost- and time-effectiveness [10-13], ability to measure "habitual" dietary intake over a relative long period [10-13] and to provide reasonable estimates of average energy and nutrient intake.

However, despite considerable advantages in terms of ease of administration and analysis, FFQ may be limited in their usefulness and, through poor design and inappropriate use, may not yield the required information [10-12]. An important limitation of this method remains the cognitive difficulty of the participants to complete the FFQ and to estimate precisely their dietary intakes using portions sizes [10-13]. Also, incomplete listing of foods in FFQ and errors in frequency lead up to inaccuracies [12]. Because of the wide variations in dietary habits within different populations and regions, a FFQ must be developed to suit specific groups and culturally distinct regions [11].

Validation of the FFQ method is important to assess the degree to which the questionnaire measures items (foods or nutrients) for which it has been designed $[12,13]$. However, there are different validation studies to assess the level of measurement error associated with use of the FFQ [11-13]. Some studies compared the FFQ results with weighed records, or diet records as reference method and other with 24-hour recalls [11-13].

The most commonly used reference methods are the dietary record or successive 24-hour diet recalls. These two open-ended dietary methods collect a variety of detailed information about food consumed over a specific period [10-12]. Nevertheless, all nutritional assessment methods have their own limitations [13]. In food records, participants may alter their diets according to their perception or even to simplify their records and there is a large burden imposed on respondents [10-12], in 24h recall, the main limitation is high investigator cost and also that participants may not report their food consumption accurately, it depends on knowledge, on respondents' short-term memory, on socially desirable answers, and on the skills of a well-trained interviewer to minimize recall bias [10-12].

In epidemiological studies, it is recommended to combine dietary methods to overcome possible sources of error and to obtain more accurate estimates of dietary intakes than that of individually methods [11-13]. The most popular combination is between the FFQ and multiple $24 \mathrm{~h}$ diet recall. This combination was superior in estimating some nutrients and foods and also enables to estimate and remove the effects of within-person variation in dietary intake [11- 13].

The need to assess the rapid changes in dietary habits in the Mediterranean area over recent years ensuing from diet transition to more westernized dietary patterns and the lack of data in Lebanon urged us to perform individual dietary surveys in this country. To fulfill this objective, we developed a food frequency questionnaire, presently not available for the specific traditional Lebanese diet. A FFQ with food photographs for portion estimation was designed to assess the habitual dietary intake of food and nutrients from traditional Lebanese dishes. Also, we decided to validate the FFQ by using a weekly dietary recall (WDR) as the reference method instead of 24-hour diet recalls because the traditional food are consumed weekly and not daily.

The purpose of the present study was to develop and validate a FFQ and to compare it with a weakly dietary recall for assessing the habitual intake of traditional Lebanese dishes in a sample representative of the Lebanese adult's population.

\section{Materials and Method}

\subsection{Design of the Food Frequency Questionnaire}

\subsubsection{Development of a Dish List}

The appropriateness of the traditional Lebanese dishes list was crucial in the food frequency method. To develop a culture-specific food list, we couldn't adapt a list based on previous surveys because traditional Lebanese dishes are not adequately represented in dietary Lebanese instrument. Initially, to define a closed ended-list of traditional dishes locally consumed by the Lebanese population and to obtain information on preferences regarding home-prepared dishes, we carried out a first preliminary $24 \mathrm{~h}$ dietary recall on 50 Lebanese adults ( 25 males, 25 females). In a second step, we compiled all the dishes mentioned in their recall, and added the missing ones using popular Lebanese cookbooks. In a third step, expert local dieticians completed and adjusted the final food list which included 56 traditional Lebanese dishes.

However, most FFQs have between 100-150 items [10-13] but they were not design for mixed traditional dishes.

\subsubsection{Determination of Food Groups}

We organized the traditional Lebanese dishes based on their similarities in nutrient content into 6 major food groups because there is minimal loss of information in estimating nutrient intakes using food intake categories [12]. The food groups were: Vegetables (fresh and cooked vegetables excluding potatoes), Cereals (rice, pasta, wheat, bulgur) and pastries, Legumes (lentils, brown fava beans, chickpeas), Meat (grilled lamb meat and poultry), Fish and Dairy products (labneh) (Table 1). 
A Comparison of Two Dietary Assessment Methods in a Mediterranean Lebanese Adults Population:

A Food Frequency Questionnaire and a Dietary Recall

Table 1. Food Frequency Questionnaire including Food groups and Lebanese traditional dishes

\begin{tabular}{|c|c|c|c|c|c|c|}
\hline \multirow{2}{*}{ Food Groups Lebanese Traditional Dishes } & \multicolumn{5}{|c|}{ Frequency } & \multirow{2}{*}{$\begin{array}{c}\text { Portion } \\
\text { Photo }\end{array}$} \\
\hline & Day & Week & Month & Year & Never & \\
\hline \multicolumn{7}{|l|}{ Total Vegetables } \\
\hline \multicolumn{7}{|l|}{ Vegetables without meat } \\
\hline \multicolumn{7}{|l|}{ Tabbouleh } \\
\hline \multicolumn{7}{|l|}{ Fattouch } \\
\hline \multicolumn{7}{|l|}{ Salatit bakle } \\
\hline \multicolumn{7}{|l|}{ Salatit roka } \\
\hline \multicolumn{7}{|l|}{ Salatit zaatar } \\
\hline \multicolumn{7}{|l|}{ Loubieh bi zayt } \\
\hline \multicolumn{7}{|l|}{ Bamia bi banadoura } \\
\hline \multicolumn{7}{|l|}{ Hindbeh b zeit } \\
\hline \multicolumn{7}{|l|}{ Msaqaa } \\
\hline \multicolumn{7}{|l|}{ Silk } \\
\hline \multicolumn{7}{|l|}{ Baba ghannouj } \\
\hline \multicolumn{7}{|l|}{ Warak anab b zayt } \\
\hline Batinjane miqli & & & & & & \\
\hline Vegetables with meat & & & & & & \\
\hline Yachnet Bazella & & & & & & \\
\hline Yachnet Sbanegh & & & & & & \\
\hline Yachnet Loubieh & & & & & & \\
\hline Molokhieh & & & & & & \\
\hline Daoud bacha & & & & & & \\
\hline Cheikh el mehchi & & & & & & \\
\hline Koussa mehchi & & & & & & \\
\hline Malfouf mehchi & & & & & & \\
\hline Warak anab bil lahmeh & & & & & & \\
\hline Cereals* and Pastries* & & & & & & \\
\hline Cereals* & & & & & & \\
\hline Riz mfalfal & & & & & & \\
\hline Maacaronies bil foron & & & & & & \\
\hline Bourghol bi dfine & & & & & & \\
\hline Chich barak wa rez & & & & & & \\
\hline Riz aa djej & & & & & & \\
\hline Moghrabiyeh & & & & & & \\
\hline Kebbe laqtine & & & & & & \\
\hline Kebbe labaniyeh & & & & & & \\
\hline Kebbe b siniyeh & & & & & & \\
\hline Kebbe ras bil lahmeh & & & & & & \\
\hline Kebbe nayeh & & & & & & \\
\hline Kafta bi siniyeh $\dagger$ & & & & & & \\
\hline Pastries* & & & & & & \\
\hline Rkakat bil jibneh & & & & & & \\
\hline Fatayer be Sabanegh & & & & & & \\
\hline Manakich b zaatar/jibneh & & & & & & \\
\hline Lahmeh baajine & & & & & & \\
\hline Pizza Lebnenyeh & & & & & & \\
\hline Samboussek b jibneh & & & & & & \\
\hline Samboussek b lahmeh & & & & & & \\
\hline Legumes & & & & & & \\
\hline Adass bi hamod & & & & & & \\
\hline Mdardara & & & & & & \\
\hline Mjaddara & & & & & & \\
\hline Hommos bi thineh & & & & & & \\
\hline Fassoulia & & & & & & \\
\hline Foul m'dammas & & & & & & \\
\hline Falafel & & & & & & \\
\hline
\end{tabular}




\begin{tabular}{|c|c|c|c|c|c|c|}
\hline \multicolumn{5}{|c|}{ Frequency } & Portion \\
\hline Food Groups Lebanese Traditional Dishes & Day & Week & Month & Year & Never & Photo \\
\hline Fish & & & & & & \\
\hline Samkeh harra & & & & & & \\
\hline Tajen samak & & & & & & \\
\hline Sayadiyeh samak & & & & & & \\
\hline Meat and Poultry & & & & & & \\
\hline Kafta michwi & & & & & & \\
\hline Chich taouk michwi & & & & & & \\
\hline Lahmeh michwi & & & & & & \\
\hline Dairy Products & & & & & & \\
\hline Labneh & & & & & & \\
\hline Chanklich & & & & & \\
\hline
\end{tabular}

* may include some meat or some poultry, $\dagger$ include potatoes

Please indicate your usual intake of each of the following dishes per Day, Week, or Month, Year, during the past year

(For example: if you consume 2 times per week Tabbouleh, write 2 in the Week column) and choose the letter corresponding to your usual consumed portion (For example: D).

\subsubsection{Determination of Food Frequency and Food Portion Sizes}

We used 5 categories to assess the frequency of consumption (times per day, per week, per month or per year, or as never), where each participant could provide the personalized frequency of food intake in an open-ended questions, aiming at higher levels of reliability [14]. For each traditional Lebanese dishes, participants indicated their average frequency of consumption over the past year (Table 1). To estimate portion size of food consumed we complemented the questionnaire with color food photographs of 3 differently sized portions of traditional Lebanese dishes $[15,16]$. Participants could choose one of the amounts among 7 proposals ( 3 shown in photographs and 4 intermediary virtual portions) to select their own portion size. Food photographs were used to improve estimations of portion size. However, due to the fact that the same photographs were used in the FFQ and in the WDR, it is possible that some errors in portion size estimation may be correlated.

\subsubsection{Pilot Study}

At the end of the development of the FFQ, we carry out a second preliminary survey on 50 other Lebanese adults, (25 males, 25 females) to test the pre-final version of the study material with the aim to check clarity, comprehensibility, acceptability and to optimize the final FFQ.

\subsection{Evaluation of the Food Frequency Questionnaire}

\subsubsection{Study Population}

566 Lebanese, 280 males (49.5\%) and 286 females (50.5\%), aged 20-62 years participated in the final study. The mean age of the total sample was $40.57 \pm 15.45$ (women: $41.18 \pm 15.06$; men $39.95 \pm 15.86$ ). The adults were selected randomly for this cross-sectional study from 6 Lebanese administrative regions (Beirut,
Mount-Lebanon, North, South, Bekaa, Nabatieh) to be representative of the global population; their socio-economical and geographical distribution was similar to that of the National Survey of Household Living Conditions 2004 [17]. Of the 679 participants initially recruited, 90 who didn't have time refused to participate in the survey, 13 who had chronic disease affecting their dietary habits and nutritional status and 10 who had food allergies were excluded. The participation rate was $80 \%$. This study received ethics clearance by the Lebanese University, and all participants signed a consent form before taking part in the study.

\subsubsection{Reference Method}

The most commonly used reference methods are the dietary record or successive 24-hour diet recalls [10-12]. We selected the diet recall as reference method because the diet recall is a rapid method, less expensive method and has relatively low respondent burden compared to dietary record [10-12]. We decided to validate the FFQ by using a weekly dietary recall (WDR) as the reference method instead of 24-hour diet recalls because the traditional food are consumed weekly and not daily.

\subsubsection{Study Design and Data Collection}

The study of the validity of the FFQ was carried out by trained nutritionists in 6 Lebanese administrative regions (Beirut, Mount-Lebanon, North, South, Bekaa, Nabatieh). Of 566 Lebanese adults visited, 126 were at work, 252 at home and 188 at universities. Information on traditional food intakes and nutrients intakes of each participant was collected by means of a FFQ which was validated using a WDR as the reference method. The FFQ was administrated before the WDR through face-to-face interviews on the same day, aiming at higher level of quality and accuracy of data collection [14].

In the FFQ, all participants were asked to estimate the number of times per day, week, month or year, they 
consumed the 56 traditional mixed dishes the year before. In the WDR, a checklist of the same 56 traditional mixed dishes was part of the interview. All participants indicated all the traditional mixed dishes they consumed during the week prior to the interview. The amount consumed was estimated for FFQ and WDR by using the same food photographs book developed and validated specially for estimating food portions sizes of Lebanese traditional dishes $[15,16]$. Each FFQ interview lasted about 50-60 minutes and the WDR interviews lasted about 20 minutes.

\subsubsection{Food Composition Database}

The food composition database that we found for Lebanon was 48 years old [18]. Therefore we could not use the Lebanese food composition database to estimate nutrient intake from the FFQ. We created using the software NutriLog, a Lebanese food composition database based on the old Lebanese [18] and USDA nutrient databases [19]. We entered local recipes of the 56 Lebanese traditional mixed dishes into the software NutriLog to obtain the nutrient content of each dish.

\subsubsection{Processing the Data}

The daily frequency of consumption by food was multiplied by the appropriate portion size in grams to obtain the daily food intake (g/day). The daily food group intake (g/day) was computed by adding the daily food intake of all food items belonging to this food category. The nutrient composition of each food was obtained from the food composition database especially designed to accommodate the nutritional composition of the traditional Lebanese dishes. The daily nutrient intake was computed by adding the daily nutrient composition of all food items.

\subsection{Statistical Analysis}

Statistical analyses were performed with the Statistical Package for the Social Sciences (SPSS) version 18.0 (SPSS Inc, Chicago, IL, USA) for men and women separately. Since food group and nutrient intakes were generally skewed towards higher values, we chose to use non-parametric statistics. To compare absolute food group intakes and nutrient intakes of traditional Lebanese dishes estimated by the FFQ and the WDR, means and standard deviation (SD), sample medians and 25th and 75th percentiles were computed. The Wilcoxon signed rank test was used to test whether the differences in distributions were statistically significant, defined as two-sided P-values $<0.05$.

Spearman correlation coefficients between the food group intakes and nutrient intakes based on the questionnaire and those based on the WDR were used as a measure of the relative validity of the ranking of participants.

To demonstrate the pattern of agreement between FFQ and WDR, we cross-classified the intakes values from both methods into thirds. We calculated the percentage of participants categorized into the same third and the extreme opposite third of intake by the FFQ and WDR.

\section{Results}

\subsection{Absolute Agreement}

Tables $2 \mathrm{a}, 2 \mathrm{~b}$ and Tables $3 \mathrm{a}, 3 \mathrm{~b}$ show respectively, for men and women, the daily food groups' intakes and nutrient intakes from the FFQ and WDR, and the median difference in percentage between the two methods. Intakes were significantly higher $(\mathrm{P}<0.05)$ with the FFQ than with the WDR for most food groups and nutrients, in both sexes. Cereals and pastries were underestimated in men (Table 2a).

Although the two methods gave similar intakes for pastries, legumes, (Table 2a), protein, SFA and magnesium in men (Table 3a) and for vegetables without meat (Table 2b), PUFA, cholesterol, vitamin E and thiamin in women (Table 3b).

The largest median relative differences between the methods were for fish in both sexes (Table 2a, 2b), for meat and poultry (Table 2a), retinol (Table $3 \mathrm{a}$ ) in men and legumes (Table 2b), riboflavin (Table $3 b$ ) in women. In total, (men and women together), the medians estimated based on the FFQ from all food groups' intakes (Tables 2a, $2 \mathrm{~b}$ ) and from all nutrient intakes (Tables $3 \mathrm{a}, 3 \mathrm{~b}$ ) were respectively $10 \%$ and $7 \%$ higher than the WDR intakes. 
Table 2a. Comparison of FFQ and WDR based on \% Median differences of daily food groups intakes from traditional Lebanese dishes in Lebanese men $(n=280)$

\begin{tabular}{|c|c|c|c|c|c|c|c|c|c|c|c|}
\hline $\begin{array}{l}\text { Food groups } \\
\text { (g/day) }\end{array}$ & $\begin{array}{l}\text { FFQ } \\
\text { Mean }\end{array}$ & $\begin{array}{l}\text { FFQ } \\
\text { SD }\end{array}$ & $\begin{array}{c}\text { FFQ } \\
\text { Median }\end{array}$ & $\begin{array}{l}\text { FFQ } \\
\text { P25 }\end{array}$ & $\begin{array}{l}\text { FFQ } \\
\text { P75 }\end{array}$ & $\begin{array}{l}\text { WDR } \\
\text { Mean }\end{array}$ & $\begin{array}{c}\text { WDR } \\
\text { SD }\end{array}$ & $\begin{array}{l}\text { WDR } \\
\text { Median }\end{array}$ & $\begin{array}{l}\text { WDR } \\
\text { P25 }\end{array}$ & $\begin{array}{l}\text { WDR } \\
\text { P75 }\end{array}$ & $\begin{array}{c}\text { Difference } \\
(\%) \\
\text { Median }\end{array}$ \\
\hline $\begin{array}{c}\text { Total } \\
\text { Vegetables }\end{array}$ & 263.2 & 129.9 & 238.5 & 178.0 & 319.4 & 229.7 & 142.5 & $204.4 *$ & 122.3 & 314.7 & 16.7 \\
\hline $\begin{array}{c}\text { Vegetables } \\
\text { without meat }\end{array}$ & 156.5 & 100.9 & 133.1 & 86.5 & 202.1 & 131.8 & 92.2 & $113.4^{*}$ & 64.7 & 186.7 & 17.4 \\
\hline $\begin{array}{l}\text { Vegetables } \\
\text { with meat }\end{array}$ & 106.7 & 59.7 & 89.9 & 65.3 & 131.3 & 97.9 & 94.4 & $75.7 *$ & 19.4 & 154.8 & 18.8 \\
\hline $\begin{array}{c}\text { Cereals and } \\
\text { pastries }\end{array}$ & 262.6 & 150.0 & 237.3 & 146.0 & 363.8 & 325.5 & 168.7 & $297.8 *$ & 194.2 & 431.2 & -20.3 \\
\hline Cereals & 202.5 & 123.8 & 176.0 & 109.4 & 290.0 & 178.7 & 94.6 & $161.8^{*}$ & 114.0 & 222.0 & 8.8 \\
\hline Pastries & 60.2 & 51.2 & 53.1 & 21.4 & 93.6 & 62.9 & 52.3 & 49.3 & 30.1 & 80.1 & 7.7 \\
\hline Legumes & 96.8 & 83.7 & 77.1 & 29.7 & 137.1 & 88.5 & 58.5 & 71.7 & 47.7 & 115.2 & 7.5 \\
\hline $\begin{array}{c}\text { Meat and } \\
\text { poultry }\end{array}$ & 61.0 & 55.7 & 57.0 & 0.0 & 96.9 & 54.2 & 43.7 & $44.0^{*}$ & 13.2 & 89.6 & 29.5 \\
\hline Fish & 9.4 & 13.4 & 4.0 & 1.1 & 12.7 & 8.4 & 13.9 & $2.9^{*}$ & 0.9 & 11.8 & 37.9 \\
\hline $\begin{array}{c}\text { Dairy } \\
\text { products }\end{array}$ & 139.5 & 93.8 & 132.3 & 62.9 & 220.6 & 129.2 & 81.9 & $125.7 *$ & 60.0 & 220.6 & 5.2 \\
\hline
\end{tabular}

Table 2b. Comparison of FFQ and WDR based on \% Median differences of daily food groups intakes from traditional Lebanese dishes in Lebanese women $(n=286)$

\begin{tabular}{|c|c|c|c|c|c|c|c|c|c|c|c|}
\hline $\begin{array}{l}\text { Food groups } \\
\text { (g/day) }\end{array}$ & $\begin{array}{l}\text { FFQ } \\
\text { Mean }\end{array}$ & $\begin{array}{l}\text { FFQ } \\
\text { SD }\end{array}$ & $\begin{array}{c}\text { FFQ } \\
\text { Median }\end{array}$ & $\begin{array}{l}\text { FFQ } \\
\text { P25 }\end{array}$ & $\begin{array}{l}\text { FFQ } \\
\text { P75 }\end{array}$ & $\begin{array}{l}\text { WDR } \\
\text { Mean }\end{array}$ & $\begin{array}{c}\text { WDR } \\
\text { SD }\end{array}$ & $\begin{array}{c}\text { WDR } \\
\text { Median }\end{array}$ & $\begin{array}{l}\text { WDR } \\
\text { P25 }\end{array}$ & $\begin{array}{l}\text { WDR } \\
\text { P75 }\end{array}$ & $\begin{array}{c}\text { Difference } \\
(\%) \\
\text { Median } \\
\end{array}$ \\
\hline $\begin{array}{c}\text { Total } \\
\text { Vegetables }\end{array}$ & 176.1 & 108.7 & 156.7 & 88.6 & 237.4 & 153.5 & 81.1 & $130.8^{*}$ & 94.1 & 197.6 & 19.8 \\
\hline $\begin{array}{c}\text { Vegetables } \\
\text { without } \\
\text { meat }\end{array}$ & 111.3 & 88.2 & 85.6 & 54.3 & 138.4 & 96.5 & 41.4 & 82.4 & 70.7 & 116.9 & 3.9 \\
\hline $\begin{array}{l}\text { Vegetables } \\
\text { with meat }\end{array}$ & 64.8 & 50.6 & 47.1 & 28.2 & 93.1 & 57.1 & 60.3 & $42.8^{*}$ & 0.0 & 87.9 & 10.0 \\
\hline $\begin{array}{c}\text { Cereals and } \\
\text { pastries }\end{array}$ & 188.1 & 102.7 & 169.1 & 114.7 & 247.3 & 162.7 & 79.2 & $153.3 *$ & 102.0 & 207.6 & 10.3 \\
\hline Cereals & 132.7 & 80.4 & 119.1 & 76.6 & 173.4 & 120.9 & 66.1 & $113.9^{*}$ & 71.4 & 159.6 & 4.6 \\
\hline Pastries & 55.3 & 47.1 & 42.9 & 23.0 & 75.8 & 41.9 & 27.3 & $40.0 *$ & 23.6 & 57.7 & 7.2 \\
\hline Legumes & 60.4 & 55.8 & 43.7 & 23.0 & 80.3 & 46.2 & 46.7 & $35.8 *$ & 11.1 & 66.0 & 22.1 \\
\hline $\begin{array}{c}\text { Meat and } \\
\text { poultry }\end{array}$ & 42.4 & 44.6 & 28.9 & 14.5 & 58.8 & 33.8 & 32.9 & $27.9^{*}$ & 0.0 & 57.4 & 3.6 \\
\hline Fish & 2.8 & 9.7 & 1.9 & 0.5 & 7.3 & 1.7 & 19.6 & $1.4 *$ & 0.0 & 31.9 & 35.7 \\
\hline $\begin{array}{c}\text { Dairy } \\
\text { products }\end{array}$ & 85.5 & 66.3 & 65.9 & 25.6 & 128.5 & 73.32 & 60.15 & $57.7 *$ & 21.2 & 128.4 & 14.2 \\
\hline
\end{tabular}

FFQ - food frequency questionnaire; S.D- Standard Deviation; WDR - weekly dietary recall; $\mathrm{P}_{25}-25$ th percentile; $\mathrm{P}_{75}-75$ th percentile

Median difference $=[($ median FFQ - median WDR $) /$ median WDR $]$ x 100; Significantly different from FFQ, Wilcoxon signed rank test: *, P<0.05

Table 3a. Comparison of FFQ and WDR based on \% Median differences of daily nutrients intakes from traditional Lebanese dishes in Lebanese men $(n=280)$

\begin{tabular}{|c|c|c|c|c|c|c|c|c|c|c|c|}
\hline $\begin{array}{l}\text { Nutrients } \\
\text { (units/day) }\end{array}$ & $\begin{array}{l}\text { FFQ } \\
\text { Mean }\end{array}$ & $\begin{array}{c}\text { FFQ } \\
\text { SD }\end{array}$ & $\begin{array}{c}\text { FFQ } \\
\text { Median }\end{array}$ & $\begin{array}{l}\text { FFQ } \\
\text { P25 }\end{array}$ & $\begin{array}{l}\text { FFQ } \\
\text { P75 }\end{array}$ & $\begin{array}{l}\text { WDR } \\
\text { Mean }\end{array}$ & $\begin{array}{c}\text { WDR } \\
\text { SD }\end{array}$ & $\begin{array}{c}\text { WDR } \\
\text { Median }\end{array}$ & $\begin{array}{l}\text { WDR } \\
\text { P25 }\end{array}$ & $\begin{array}{l}\text { WDR } \\
\text { P75 }\end{array}$ & $\begin{array}{c}\text { Difference } \\
(\%) \\
\text { Median }\end{array}$ \\
\hline Energy (kJ) & 4244.9 & 2264.2 & 3685.5 & 2610.8 & 5458.0 & 4025.2 & 2064.2 & $3646.1^{*}$ & 2538.6 & 5203.1 & 1.1 \\
\hline $\begin{array}{c}\text { Carbohydrate } \\
\text { (g) }\end{array}$ & 68.0 & 38.5 & 58.6 & 41.2 & 84.0 & 64.7 & 37.1 & $54.2 *$ & 38.8 & 81.6 & 8.1 \\
\hline Protein (g) & 36.3 & 22.7 & 31.3 & 20.3 & 46.5 & 36.0 & 22.8 & 31.8 & 19.7 & 46.9 & -1.6 \\
\hline Fat $(\mathrm{g})$ & 65.7 & 35.2 & 60.0 & 41.7 & 83.5 & 62.8 & 34.1 & $55.9 *$ & 39.3 & 77.3 & 7.3 \\
\hline MUFA (g) & 30.7 & 18.2 & 27.6 & 19.3 & 35.9 & 28.9 & 17.3 & $25.1 *$ & 18.0 & 35.8 & 10.0 \\
\hline PUFA (g) & 16.3 & 10.3 & 13.6 & 9.0 & 21.4 & 15.3 & 9.9 & $13.4^{*}$ & 7.6 & 20.3 & 1.5 \\
\hline SFA (g) & 16.1 & 10.1 & 14.4 & 9.4 & 19.5 & 15.8 & 10.2 & 13.3 & 8.7 & 20.5 & 8.3 \\
\hline $\begin{array}{c}\text { Cholesterol } \\
(\mathrm{mg})\end{array}$ & 93.7 & 125.8 & 67.4 & 37.6 & 113.7 & 85.4 & 124.8 & $57.4 *$ & 29.1 & 103.0 & 17.4 \\
\hline $\begin{array}{l}\text { Dietary fiber } \\
(\mathrm{g})\end{array}$ & 10.6 & 5.5 & 9.2 & 7.1 & 13.0 & 10.0 & 5.2 & $9.0^{*}$ & 6.5 & 12.7 & 2.2 \\
\hline
\end{tabular}




\begin{tabular}{|c|c|c|c|c|c|c|c|c|c|c|c|}
\hline $\begin{array}{c}\text { Nutrients } \\
(\text { units/day) }\end{array}$ & $\begin{array}{c}\text { FFQ } \\
\text { Mean }\end{array}$ & $\begin{array}{c}\text { FFQ } \\
\text { SD }\end{array}$ & $\begin{array}{c}\text { FFQ } \\
\text { Median }\end{array}$ & $\begin{array}{c}\text { FFQ } \\
\text { P25 }\end{array}$ & $\begin{array}{c}\text { FFQ } \\
\text { P75 }\end{array}$ & $\begin{array}{c}\text { WDR } \\
\text { Mean }\end{array}$ & $\begin{array}{c}\text { WDR } \\
\text { SD }\end{array}$ & $\begin{array}{c}\text { WDR } \\
\text { Median }\end{array}$ & $\begin{array}{c}\text { WDR } \\
\text { P25 }\end{array}$ & $\begin{array}{c}\text { WDR } \\
\text { P75 }\end{array}$ & $\begin{array}{c}\text { Difference } \\
(\%) \\
\text { Median }\end{array}$ \\
\hline Retinol $(\mu \mathrm{g})$ & 246.1 & 245.6 & 180.3 & 92.7 & 306.7 & 232.7 & 232.9 & $147.7^{*}$ & 78.8 & 296.6 & 22.1 \\
\hline \begin{tabular}{c}
\hline -carotene \\
$(\mu \mathrm{g})$
\end{tabular} & 1963.5 & 2071.9 & 1524.5 & 887.3 & 2353.1 & 1860.8 & 2075.9 & $1330.5^{*}$ & 839.9 & 2140.3 & 14.6 \\
\hline $\begin{array}{c}\text { Vitamin E } \\
(\mathrm{mg})\end{array}$ & 16.0 & 12.7 & 12.3 & 8.1 & 20.0 & 14.5 & 12.5 & $10.9^{*}$ & 7.1 & 17.4 & 12.8 \\
\hline $\begin{array}{c}\text { Vitamin } \mathrm{C} \\
(\mathrm{mg})\end{array}$ & 74.4 & 48.9 & 61.5 & 41.1 & 94.9 & 68.2 & 44.7 & $56.6^{*}$ & 37.2 & 89.9 & 8.7 \\
\hline Thiamin $(\mathrm{mg})$ & 0.6 & 0.5 & 0.5 & 0.3 & 0.7 & 0.6 & 0.5 & $0.4^{*}$ & 0.3 & 0.7 & 25.0 \\
\hline $\begin{array}{c}\text { Riboflavin } \\
(\mathrm{mg})\end{array}$ & 0.5 & 0.4 & 0.4 & 0.3 & 0.6 & 0.5 & 0.4 & $0.4^{*}$ & 0.3 & 0.6 & 0.0 \\
\hline Folate $(\mu \mathrm{g})$ & 170.3 & 162.3 & 125.9 & 83.3 & 199.6 & 160.2 & 159.8 & $118.1 *$ & 82.9 & 187.6 & 6.6 \\
\hline Calcium $(\mathrm{mg})$ & 306.4 & 186.1 & 250.8 & 176.3 & 400.2 & 292.8 & 183.4 & $256.0^{*}$ & 167.1 & 367.6 & -2.0 \\
\hline $\begin{array}{c}\text { Magnesium } \\
(\mathrm{mg})\end{array}$ & 165.8 & 95.0 & 147.5 & 99.8 & 207.2 & 161.2 & 99.0 & 140.3 & 100.1 & 202.4 & 5.1 \\
\hline Iron $(\mathrm{mg})$ & 10.2 & 5.8 & 9.2 & 6.5 & 12.8 & 9.6 & 5.5 & $8.8^{*}$ & 5.7 & 12.0 & 4.5 \\
\hline $\begin{array}{c}\text { Potassium } \\
(\mathrm{mg})\end{array}$ & 1300.1 & 626.5 & 1194.3 & 866.8 & 1623.9 & 1190.1 & 564.4 & $1085.8^{*}$ & 777.6 & 1462.5 & 10.0 \\
\hline Sodium (mg) & 1840.9 & 1005.2 & 1657.5 & 1160.7 & 2220.1 & 1677.0 & 952.1 & $1554.5^{*}$ & 1006.4 & 2157.5 & 6.6 \\
\hline
\end{tabular}

Table 3b. Comparison of FFQ and WDR based on \% Median differences of daily nutrients intakes from traditional Lebanese dishes in Lebanese women $(n=286)$

\begin{tabular}{|c|c|c|c|c|c|c|c|c|c|c|c|}
\hline $\begin{array}{l}\text { Nutrients } \\
\text { (units/day) }\end{array}$ & $\begin{array}{l}\text { FFQ } \\
\text { Mean }\end{array}$ & $\begin{array}{c}\text { FFQ } \\
\text { SD }\end{array}$ & $\begin{array}{c}\text { FFQ } \\
\text { Median }\end{array}$ & $\begin{array}{l}\text { FFQ } \\
\text { P25 }\end{array}$ & $\begin{array}{l}\text { FFQ } \\
\text { P75 }\end{array}$ & $\begin{array}{l}\text { WDR } \\
\text { Mean }\end{array}$ & $\begin{array}{l}\text { WDR } \\
\text { SD }\end{array}$ & $\begin{array}{l}\text { WDR } \\
\text { Median }\end{array}$ & $\begin{array}{l}\text { WDR } \\
\text { P25 }\end{array}$ & $\begin{array}{l}\text { WDR } \\
\text { P75 }\end{array}$ & $\begin{array}{c}\text { Difference } \\
(\%) \\
\text { Median } \\
\end{array}$ \\
\hline Energy (kJ) & 2738.7 & 1631.2 & 2267.2 & 1495.1 & 3628.6 & 2474.5 & 1194.4 & $2217.9 *$ & 1682.2 & 3165.8 & 2.2 \\
\hline $\begin{array}{c}\text { Carbohydrate } \\
(\mathrm{g})\end{array}$ & 44.7 & 28.5 & 37.8 & 22.7 & 58.4 & 39.7 & 21.0 & $36.9 *$ & 25.8 & 50.7 & 2.4 \\
\hline Protein (g) & 24.3 & 15.7 & 19.9 & 12.3 & 33.1 & 21.7 & 13.5 & $19.0 *$ & 11.7 & 27.9 & 4.7 \\
\hline Fat (g) & 45.1 & 29.0 & 38.6 & 23.9 & 58.7 & 40.1 & 21.8 & $36.9^{*}$ & 24.7 & 50.7 & 4.6 \\
\hline MUFA (g) & 20.5 & 13.3 & 18.1 & 10.6 & 26.9 & 18.3 & 9.6 & $16.6^{*}$ & 11.5 & 22.6 & 9.0 \\
\hline PUFA (g) & 11.2 & 8.2 & 8.9 & 5.6 & 14.2 & 10.2 & 6.3 & 8.8 & 5.9 & 13.7 & 1.1 \\
\hline SFA (g) & 10.7 & 7.0 & 9.4 & 5.4 & 13.5 & 9.2 & 4.9 & $8.1^{*}$ & 5.9 & 11.3 & 16.0 \\
\hline $\begin{array}{c}\text { Cholesterol } \\
(\mathrm{mg})\end{array}$ & 44.6 & 38.1 & 33.0 & 18.8 & 58.7 & 43.3 & 28.7 & 38.2 & 23.8 & 56.0 & -13.6 \\
\hline $\begin{array}{c}\text { Dietary fiber } \\
(\mathrm{g})\end{array}$ & 6.8 & 4.0 & 6.1 & 3.7 & 8.7 & 6.2 & 2.9 & $5.6^{*}$ & 4.1 & 7.6 & 8.9 \\
\hline Retinol $(\mu \mathrm{g})$ & 168.9 & 180.4 & 112.5 & 60.5 & 210.9 & 151.3 & 142.7 & $104.4^{*}$ & 49.6 & 205.4 & 7.8 \\
\hline $\begin{array}{c}\beta \text {-carotene } \\
(\mu \mathrm{g})\end{array}$ & 1231.1 & 1058.4 & 925.0 & 473.6 & 1523.6 & 1106.7 & 829.3 & $936.0 *$ & 544.9 & 1341.3 & -1.2 \\
\hline $\begin{array}{c}\text { Vitamin E } \\
(\mathrm{mg})\end{array}$ & 9.2 & 7.3 & 7.2 & 4.1 & 11.8 & 8.7 & 5.7 & 7.0 & 4.7 & 11.0 & 2.9 \\
\hline $\begin{array}{c}\text { Vitamin C } \\
(\mathrm{mg})\end{array}$ & 47.3 & 32.3 & 40.2 & 25.7 & 60.3 & 43.2 & 23.4 & $37.5^{*}$ & 26.4 & 56.0 & 7.2 \\
\hline Thiamin (mg) & 0.4 & 0.3 & 0.3 & 0.2 & 0.4 & 0.3 & 0.2 & 0.3 & 0.2 & 0.4 & 0.0 \\
\hline $\begin{array}{c}\text { Riboflavin } \\
(\mathrm{mg})\end{array}$ & 0.3 & 0.2 & 0.3 & 0.2 & 0.4 & 0.3 & 0.1 & $0.2^{*}$ & 0.2 & 0.3 & 50.0 \\
\hline Folate $(\mu \mathrm{g})$ & 88.7 & 51.7 & 78.0 & 52.4 & 113.9 & 80.8 & 40.1 & $70.4^{*}$ & 53.6 & 103.7 & 10.8 \\
\hline Calcium (mg) & 197.4 & 121.0 & 167.4 & 111.5 & 257.3 & 178.3 & 93.6 & $159.4^{*}$ & 112.7 & 222.6 & 5.0 \\
\hline $\begin{array}{l}\text { Magnesium } \\
(\mathrm{mg})\end{array}$ & 114.1 & 70.5 & 99.2 & 61.3 & 148.5 & 99.7 & 53.2 & $93.7 *$ & 61.6 & 126.0 & 5.9 \\
\hline Iron (mg) & 6.7 & 4.0 & 5.9 & 3.9 & 8.5 & 6.0 & 3.0 & $5.3 *$ & 3.9 & 7.5 & 11.3 \\
\hline $\begin{array}{c}\text { Potassium } \\
(\mathrm{mg})\end{array}$ & 835.7 & 471.0 & 749.9 & 503.1 & 1043.2 & 743.6 & 327.9 & $689.1^{*}$ & 504.5 & 932.6 & 8.8 \\
\hline Sodium (mg) & 1132.5 & 651.7 & 1001.1 & 643.0 & 1459.0 & 1029.1 & 486.8 & $932.8 *$ & 684.2 & 1279.2 & 7.3 \\
\hline
\end{tabular}

FFQ - food frequency questionnaire; WDR - weekly dietary recall; S.D- Standard Deviation; $\mathrm{P}_{25}-25$ th percentile; $\mathrm{P}_{75}-75$ th percentile;

MUFA - monounsaturated fatty acids; PUFA - polyunsaturated fatty acids; SFA - saturated fatty acids

Median difference $=[($ median FFQ - median WDR $) /$ median WDR $] \times 100$; Significantly different from FFQ, Wilcoxon signed rank test: *, P $<0.05$ 


\subsection{Relative Agreement}

Tables 4 and 5 show respectively, for men and women, the Spearman correlation coefficients calculated for each food group intakes and nutrient intakes. Median Spearman correlation coefficients based on FFQ and WDR were 0.54 in men and 0.53 in women for all food groups estimates (Table 4), and 0.78 in men and 0.67 in women for all nutrients estimates (Table 5). Spearman correlation coefficients ranged from 0.22 for vegetables with meat to 0.95 for cereals, pastries and dairy products in men and from 0.30 for fish to 0.86 for dairy products in women (Table 4). Spearman correlation coefficients ranged from 0.66 for retinol to 0.87 for folate in men and from 0.58 for PUFA to 0.69 for energy, fat, folate and potassium in women (Table 5).
For all food groups (Table 4), a median of 53\% of men was correctly classified (range 39\% for vegetables with meat to $92 \%$ for fish), and between 0 and $15 \%$ of men (median 7\%) were grossly misclassified. For all food groups (Table 4), a median of 54\% of women was correctly classified (range $39 \%$ for total vegetables to $79 \%$ for dairy products), and between 2 and $23 \%$ of women (median $8 \%$ ) were grossly misclassified.

For all nutrients (Table 5), a median of $64 \%$ of men was correctly classified (range 59\% for PUFA and retinol to $75 \%$ for cholesterol and folate), and between 1 and $6 \%$ of men (median 3\%) were grossly misclassified. For all nutrients (Table 5), a median of 59\% of women was correctly classified (range $54 \%$ for PUFA to $70 \%$ for vitamin E), and between 3 and $7 \%$ of women (median 4\%) were grossly misclassified.

Table 4. Spearman correlation coefficients between FFQ and WDR and percentages of participants classified into the same and opposite thirds of food groups intakes from traditional Lebanese dishes

\begin{tabular}{|c|c|c|c|c|c|c|}
\hline \multirow[b]{2}{*}{$\begin{array}{l}\text { Food groups } \\
\text { (g/day) }\end{array}$} & \multicolumn{3}{|c|}{ Men $(n=280)$} & \multicolumn{3}{|c|}{ Women $(n=286)$} \\
\hline & $\begin{array}{c}\text { Spearman } \\
\text { correlation } \mathrm{r}\end{array}$ & $\begin{array}{c}\% \text { classified in } \\
\text { Same third }\end{array}$ & $\begin{array}{l}\% \text { classified in } \\
\text { Opposite third }\end{array}$ & $\begin{array}{c}\text { Spearman } \\
\text { correlation } \mathrm{r}\end{array}$ & $\begin{array}{c}\% \text { classified in } \\
\text { Same third }\end{array}$ & $\begin{array}{l}\% \text { classified in } \\
\text { Opposite third }\end{array}$ \\
\hline Total Vegetables & $0.38 * *$ & 47.9 & 11.4 & $0.35 * *$ & 39.5 & 11.5 \\
\hline $\begin{array}{c}\text { Vegetables without } \\
\text { meat }\end{array}$ & $0.51 * *$ & 51.8 & 7.5 & $0.40 * *$ & 47.2 & 11.2 \\
\hline $\begin{array}{c}\text { Vegetables with } \\
\text { meat }\end{array}$ & $0.22 * *$ & 39.3 & 14.6 & $0.38 * *$ & 42.0 & 10.5 \\
\hline Cereals and pastries & $0.95 * *$ & 85.0 & 0.0 & $0.55 * *$ & 59.8 & 7.0 \\
\hline Cereals & $0.49 * *$ & 52.1 & 7.9 & $0.50 * *$ & 51.7 & 9.8 \\
\hline Pastries & $0.35 * *$ & 44.6 & 11.1 & $0.59 * *$ & 56.3 & 5.6 \\
\hline Legumes & $0.57 * *$ & 54.6 & 6.1 & $0.62 * *$ & 57.7 & 5.9 \\
\hline Meat and poultry & $0.84 * *$ & 69.6 & 0.7 & $0.72 * *$ & 62.9 & 3.1 \\
\hline Fish & $0.92 * *$ & 91.8 & 1.1 & $0.30 * *$ & 43.0 & 22.7 \\
\hline Dairy products & $0.95^{* *}$ & 90.0 & 0.4 & $0.86 * *$ & 79.0 & 2.4 \\
\hline Total & $0.54 * *$ & 53.35 & 6.8 & $0.53 * *$ & 54.0 & 8.4 \\
\hline
\end{tabular}

FFQ - food frequency questionnaire; WDR - weekly dietary recall; $r$ - Spearman correlation coefficient, **Correlation is significant at the 0.01 level

Table 5. Spearman correlation coefficients between FFQ and WDR and percentages of participants classified into the same and opposite thirds of nutrients intakes from traditional Lebanese dishes

\begin{tabular}{|c|c|c|c|c|c|c|}
\hline \multirow[b]{2}{*}{$\begin{array}{l}\text { Nutrients } \\
\text { (units/day) }\end{array}$} & \multicolumn{3}{|c|}{$\operatorname{Men}(n=280)$} & \multicolumn{3}{|c|}{ Women $(n=286)$} \\
\hline & $\begin{array}{c}\text { Spearman } \\
\text { correlation } \mathrm{r}\end{array}$ & $\begin{array}{c}\% \text { classified in } \\
\text { Same third }\end{array}$ & $\begin{array}{l}\% \text { classified in } \\
\text { Opposite third }\end{array}$ & $\begin{array}{c}\text { Spearman } \\
\text { correlation } \mathrm{r}\end{array}$ & $\begin{array}{c}\% \text { classified in } \\
\text { Same third }\end{array}$ & $\begin{array}{l}\% \text { classified in } \\
\text { Opposite third }\end{array}$ \\
\hline Energy (kJ) & $0.78 * *$ & 64.3 & 2.9 & $0.69 * *$ & 61.5 & 2.8 \\
\hline Carbohydrate (g) & $0.76^{* *}$ & 67.1 & 2.9 & $0.68 * *$ & 61.2 & 4.5 \\
\hline Protein (g) & $0.77 * *$ & 64.3 & 2.1 & $0.67 * *$ & 60.1 & 3.5 \\
\hline Fat $(\mathrm{g})$ & $0.77 * *$ & 63.6 & 1.4 & $0.69 * *$ & 59.8 & 3.1 \\
\hline MUFA (g) & $0.79 * *$ & 63.6 & 2.9 & $0.65 * *$ & 55.2 & 5.6 \\
\hline PUFA (g) & $0.71 * *$ & 59.3 & 4.3 & $0.58 * *$ & 54.5 & 6.3 \\
\hline SFA (g) & $0.76^{* *}$ & 66.4 & 3.6 & $0.68 * *$ & 59.4 & 3.5 \\
\hline Cholesterol (mg) & $0.85 * *$ & 75.4 & 1.1 & $0.68 * *$ & 57.3 & 5.6 \\
\hline Dietary fiber (g) & $0.76^{* *}$ & 63.9 & 3.9 & $0.66^{* *}$ & 59.1 & 5.2 \\
\hline Retinol $(\mu \mathrm{g})$ & $0.66^{* *}$ & 59.3 & 2.9 & $0.60 * *$ & 57.7 & 6.6 \\
\hline$\beta$-carotene $(\mu \mathrm{g})$ & $0.69 * *$ & 60.7 & 5.7 & $0.66 * *$ & 57.3 & 4.2 \\
\hline Vitamin E (mg) & $0.81 * *$ & 67.1 & 1.4 & $0.64 * *$ & 61.9 & 5.9 \\
\hline
\end{tabular}




\begin{tabular}{|c|c|c|c|c|c|c|}
\hline & \multicolumn{3}{|c|}{ Men (n=280) } & \multicolumn{3}{c|}{ Women (n=286) } \\
\hline $\begin{array}{c}\text { Food groups } \\
\text { (g/day) }\end{array}$ & $\begin{array}{c}\text { Spearman } \\
\text { correlation r }\end{array}$ & $\begin{array}{c}\text { \% classified in } \\
\text { Same third }\end{array}$ & $\begin{array}{c}\text { \% classified in } \\
\text { Opposite third }\end{array}$ & $\begin{array}{c}\text { Spearman } \\
\text { correlation } \mathrm{r}\end{array}$ & $\begin{array}{c}\text { \% classified in } \\
\text { Same third }\end{array}$ & $\begin{array}{c}\text { \% classified in } \\
\text { Opposite third }\end{array}$ \\
\hline Vitamin C (mg) & $0.79^{* *}$ & 68.2 & 3.2 & $0.65^{* *}$ & 56.6 & 4.2 \\
\hline Thiamin $(\mathrm{mg})$ & $0.79^{* *}$ & 63.9 & 2.5 & $0.62^{* *}$ & 56.6 & 4.9 \\
\hline Riboflavin $(\mathrm{mg})$ & $0.83^{* *}$ & 71.8 & 2.5 & $0.68^{* *}$ & 60.5 & 3.8 \\
\hline Folate $(\mu \mathrm{g})$ & $0.87^{* *}$ & 75 & 1.4 & $0.69^{* *}$ & 61.5 & 4.2 \\
\hline Calcium $(\mathrm{mg})$ & $0.79^{* *}$ & 64.6 & 2.5 & $0.66^{* *}$ & 60.1 & 5.6 \\
\hline Magnesium $(\mathrm{mg})$ & $0.77^{* *}$ & 61.8 & 1.8 & $0.64^{* *}$ & 55.9 & 3.5 \\
\hline Iron $(\mathrm{mg})$ & $0.79^{* *}$ & 68.2 & 3.9 & $0.67^{* *}$ & 58.4 & 3.8 \\
\hline Potassium $(\mathrm{mg})$ & $0.78^{* *}$ & 69.6 & 3.9 & $0.69^{* *}$ & 60.1 & 2.8 \\
\hline Sodium $(\mathrm{mg})$ & $0.80^{* *}$ & 63.9 & 1.8 & $0.67^{* *}$ & 60.8 & 4.2 \\
\hline Total & $0.78^{* *}$ & 64.3 & 2.9 & $0.67^{* *}$ & 59.4 & 4.2 \\
\hline
\end{tabular}

FFQ - food frequency questionnaire; WDR - weekly dietary recall; MUFA - monounsaturated fatty acids; PUFA - polyunsaturated fatty acids;

SFA - saturated fatty acids; $r$ - Spearman correlation coefficient; **Correlation is significant at the 0.01 level

\section{Discussion}

Validation studies differ in terms of the demographic characteristics of their study populations, types of reference data used, sample sizes, lengths of questionnaires, and study designs [10-13]. Differences between populations make it necessary to develop culturally tools for the population in whom it is used in order to evaluate their specific dietary habits. Unfortunately, there is little information on the consumption of traditional foods in Lebanon, a Mediterranean-diet-based population. It is in this context that results from the present study are discussed.

We found a general overestimation of food group intakes estimated from the FFQ compared to the WDR excepted for cereals and pastries in men. Other validity studies of FFQ have also shown both overestimates and underestimates for food group [20-26]. Usually, FFQ overestimates most food groups than the reference method [10]. Errors in interpretation of specific items, differences in frequency and portion sizes may be lead to over reporting between FFQ and WDR [10]. There is evidence that when using 24-hour diet recalls or other methods, high consumers underestimate their intake and participants who consume little tend to overestimate (the so-called flat slope syndrome) [10].

The present results show that this FFQ provides reasonably good validity in relation to WDR for many food groups in accordance with the interpretation criteria for validation of dietary intake assessment $(r \geq 0.5)$ [27]. The Spearman correlation coefficients ranged from 0.22 to 0.95 in men (median 0.53), and from 0.30 to 0.86 in women (median 0.53) for all food groups. Our results showed that main food group correlations were very similar to those reports $[20,21,28]$ and better than those observed in other validation studies $[22-26,29,30]$. Precise comparisons are difficult, however, because of methodological differences, differences in populations and differences in food groups reported.

It is clear that, as in our study, the low relative validity for the ranking of individuals according to vegetable intake is generally fair ( $\mathrm{r}=0.38$ for men, $\mathrm{r}=0.35$ for women), similar to the results reported by Keyzer et al. [29] and Streppel et al. [25], but lower than other researches [20, 21, 23]. A small inter-individual variation in consumption frequency and measurement errors in portion size estimation might be possible reasons for the generally low relative validity for estimates of vegetable intake. Measurement error could be provided from different ways to group foods in studies, vegetables are more frequently part of cooked dishes and often sliced or cut which makes them more difficult to quantify [29].

In our study, our participant's classification in the same tertile/quartile for all the food groups was good $\geq 50 \%$ according to Lombard et al., [27]. The exact agreement by thirds of intake ranged from $39 \%$ to $92 \%$ in Lebanese men (median 53.3\%) and from 39\% to 79\% in Lebanese women (median 54\%) with both of the two methods. These results concur with other reports $[20,22,23,29]$.

We found a low frequency of gross misclassification for all food groups, between 0 and $15 \%$ in men (median $7 \%$ ) and 2 and $23 \%$ in women (median 8\%). The extreme median classification into opposite tertiles was $<10 \%$ resulting in a good validation for all food group by the FFQ and the WDR [27] and similar to other reports [20, 22, 23, 29, 30].

Analyses at the nutrient level showed that intakes were significantly higher $(\mathrm{P}<0.05)$ with the FFQ than with the WDR for most nutrients. These results are similar to the observations of other researchers [20, 22, 24, 27, 31-33]. The overestimation of the FFQ can be associated to the use of 24-hour recall as a reference dietary method related to correlated errors of bias memories in the both methods [34]. The apparent differences among validation studies in the direction (over or under-estimation of nutrient medians) and degree of bias may be explained partly by variations in 
the number and specificity of items on the questionnaire and different reference method and population $[10,32]$.

Our research indicated a high correlation level between FFQ and WDR for all nutrients, and ranged from 0.66 to 0.87 in men (median 0.78 ) and from 0.58 to 0.69 in women (median 0.67). The majority of studies validating food frequency questionnaires against diet records and recalls have reported good correlation coefficients ranging between 0.4 and 0.7 for all nutrients [10-12, 27]. Our correlation coefficient's $\geq 0.50$ reflected a strength association between the two methods according to Lombard et al. [27], and were comparable to other researches $[22,25,31,33,35,36]$. The fact that in our study, the correlation coefficients were slightly higher may be attributed to the method of administration of the FFQ, face to face interview, where the communications achieved more reliable answers than self-administrated questionnaire and helped the participants to recall better their dietary intakes $[10-12,34]$. The use of the same trained nutritionist interviewer for each participant for both methods administrated in short time period, (the same day) and food photographs of portion sizes also improved dietary estimations [10-12, 34].

For the majority of nutrients, FFQ estimates in the present study were significantly different from those of WDR, even though estimates from the two methods were correlated significantly. It is plausible that although the food frequency questionnaire overestimated medians for certain nutrients, it did so in a consistent manner. Nevertheless, the FFQ demonstrated strong correlations for all nutrients.

In this study, for all nutrients, the median percentage of participants correctly classified in the same third by both the food frequency questionnaire and the WDR range from $59 \%$ to $75 \%$ in men (median $64 \%$ ), and from $54 \%$ to $70 \%$ in women (median 59\%). According to Lombard et al. [27], our participant's classification in the in same tertile/quartile was good $\geq 50 \%$ for nutrients and comparable to other studies [20,22, 23, 31].

Our findings showed that gross misclassification was low for all nutrients in Lebanese adults, between 1 and 6\% in men (median 3\%) and between 3 and $7 \%$ in women (median 4\%). The extreme classification into opposite tertiles was $<10 \%$ resulting in a good validation for all nutrients by the FFQ and the WDR [27] and similar to other studies [20, 22, 23, 26, 31, 32].

Thus, the favorable correlations and third classifications that were obtained suggest that our instrument is suitable for ranking individuals according to their food and nutrient intakes. On the other hand, this is the first study ever done to validate a FFQ among Lebanese adults with a sample size large enough $(\mathrm{n}=566)$ to detect as statistically significant even small correlation coefficients which may be considered as minimally valid $(r>0.20)$, [27]

Our FFQ was validated in the Lebanese adult's population where it will be used. Participants had agreed to participate in the study, so strictly speaking they were volunteers. Highly motivated volunteers may increase the level of agreement with the standard [10]. However, the participants in our study did not have any particular motivation and participants from different occupations were included. They were selected to be representative of the global population.

The present study demonstrates the validity of the FFQ from only traditional Lebanese dishes and has certain limitations. We used a weekly dietary recall as reference method, instead of multiple $24 \mathrm{~h}$ recalls most widely validated and used in the literature [11,34], because traditional food are habitually consumed weekly and not daily. This could have influenced in some ways the results $[11,34]$. Due to time constraints, the FFQ and the WDR were only performed once and this could have given an overestimation of the validity. Ideally, several measures with independent days spread over a longer time period would have reflected the reproducibility of habitual dietary intake better [10, 13, 34]. However, survey repetition needs a lot of resources, time and can also influence a participants' diet by increasing the systematic error of self-report [11, 34]. Furthermore, important limitations of this dietary intake assessment method are that the participants' consumption from FFQ and WDR depends on similar recall biases; they have shared common errors, since they report retrospectively their intakes referred to a long period of time and they use the same food composition databases to convert food consumption to energy and nutrient intakes [10-13, 22, 34]. Correlations between the FFQ and the WDR may be overestimated because of correlated errors [11, 22, 34]. Major sources of error of the FFQs could be occurred by restrictions caused by a fixed list of foods, interpretation of questions and perception of serving sizes $[10,13]$.

Nevertheless, this FFQ was in-line with other Mediterranean validations studies' [20, 31, 22, 23, 29], suggesting that the FFQ of traditional Lebanese diet intake would be comparable to assessments with repeatability's.

\section{Conclusions}

The results of the present study reported that the FFQ is well-correlated with the WDR for assessing the habitual dietary and nutritional intakes of traditional Lebanese dishes in a sample representative of the Lebanese adult's population. This novel dish-based FFQ was specifically designed for the Mediterranean culture. The FFQ, was found to have an acceptable level of validity compared with the reference method, and may be a useful instrument to rank individuals according to their usual consumption of traditional foods and nutrients. More researches are needed to complete the validation study in other population like children, adolescents and elderly groups and the reproducibility across all age groups. In future 
researches, this new tool will help to estimate the contribution of Mediterranean Lebanese traditional meals to the daily diet and also to monitor the nutritional status of Lebanese adults' population in order to prevent diseases occurrence in relation with more westernized dietary patterns. Therefore, this instrument can be used to track changes in traditional Mediterranean diet resulting from diet transition, in order to support better quality dietary intake and to facilitate nutritional education and health promotion. In conclusion, our validated tool for the Lebanese adult Mediterranean population could serve other researchers to assess their specific traditional dietary habits.

\section{Acknowledgements}

We are grateful to the nutritionists who collected the dietary data in Lebanon. We are grateful to the participants of the study for their cooperation. This study was carried out by the Lebanese University.

\section{REFERENCES}

[1] L. M. Donini, L. Serra-Majem, M. Bulló, Á. Gil, J. Salas-Salvadó, The Mediterranean diet: Culture, health and science, British Journal of Nutrition, Vol.113, No.Suppl. 2, S1-S3, 2015.

[2] M.A. Martinez-Gonzalez, N. Martin-Calvo, Mediterranean diet and life expectancy; beyond olive oil, fruits, and vegetables, Current Opinion in Clinical Nutrition and Metabolic Care, Vol.19, 401-7, 2016

[3] M. Guasch-Ferré, J. Salas-Salvadó, E. Ros, R. Estruch, D. Corella, M. Fitó, M.A. Martínez-González , for the PREDIMED Investigators, The PREDIMED trial, Mediterranean diet and health outcomes: How strong is the evidence?, Nutrition, Metabolism \& Cardiovascular Diseases Vol.27, 624-632, 2017.

[4] M. Dinu, G. Pagliai, A. Casini, F.Sofi. Mediterranean diet and multiple health outcomes: an umbrella review of meta-analyses of observational studies and randomised trials. European Journal of Clinical Nutrition, Vol.72, 30-43, 2018.

[5] R. Estruch, E. Ros, J. Salas Salvado, M.I. Covas, D. Corella, F. Aros, E. Gomez Gracia, V. Ruiz Gutierrez, M. Fiol, J. Lapetra, R.M. Lamuela Raventos, L. Serra Majem, X. Pinto, J. Basora, M.A. Munoz, J.V. Sorli, J.A. Martinez, M. Fito, A. Gea, M.A. Hernan, and M.A. Martinez Gonzalez, for the PREDIMED Study Investigators, Primary Prevention of Cardiovascular Disease with a Mediterranean Diet Supplemented with Extra-Virgin Olive Oil or Nuts, The New England Journal of Medicine, Vol.378, No.25, e34(1)-e34(14), 2018.

[6] M.A. Martínez-González, J. Salas-Salvadó, R. Estruch, D. Corella, M. Fitó, E. Ros. Benefits of the Mediterranean diet: insights from the PREDIMED Study. Progress in
Cardiovasculair Diseases, Vol.58, 50-60, 2015.

[7] A. Gea, M. Bes-Rastrollo, E. Toledo, M. Garcia-Lopez, J.J Beunza, R. Estruch, M.A Martinez-Gonzalez, Mediterranean alcohol-drinking pattern and mortality in the SUN (Seguimiento Universidad de Navarra) Project: A prospective cohort study, British Journal of Nutrition, Vol.111, 1871-1880, 2014

[8] M.A. Martínez-González, M.S. Hershey, I. Zazpe, A. Trichopoulou, Transferability of the Mediterranean Diet to Non-Mediterranean Countries. What Is and What Is Not the Mediterranean Diet, Nutrients, Vol.9, No.11, E 1226, 2017.

[9] G. Middleton, R. Keegan, M.F. Smith, A. Alkhatib, M. Klonizakis. Brief Report: Implementing a Mediterranean Diet Intervention into a RCT: Lessons Learned from a NonMediterranean Based Country. The Journal of Nutrition, Health \& Aging, Vol.19, No.10, 1019-22, 2015.

[10] J.A. Lovegrove, L. Hodson, S. Sharma and S.A. Lanham-New, Nutrition Research Methodologies, First Edition, the Nutrition Society TextBook Series, Wiley Blackwell, pp. 1-359, 2015.

[11] J.S. Shim, K. Oh, H.C Kim, Dietary assessment methods in epidemiologic studies, Epidemiology and Health, Vol. 36, e2014009, 2014.

[12] F.E. Thompson \& A.F. Subar, Dietary assessment methodology. In Nutrition in the Prevention and Treatment of Disease, 2nd ed., A.M. Coulston and C.J. Boushey editors, San Diego, CA: Academic Press, pp. 5-46, 2008.

[13] A. Naska, A. Lagiou, P. Lagiou, Dietary assessment methods in epidemiological research: current state of the art and future prospects, F1000Research, Vol.6, 926, 2017.

[14] L. Serra-Majem, L.F. Andersen, P. Henríque-Sánchez, J. Doreste-Alonso, A. Sànchez-Villegas, A Ortiz-Andrelluchi, E. Negri, C. La Vecchia, Evaluating the quality of dietary intake validation studies. British Journal of Nutrition, Vol. 102, No. Suppl. 1, S3-S9, 2009.

[15] M. Tueni, A. Mounayar, and I. Birlouez-Aragon, Design and validation of a colour food photography booklet for estimating food portion size in the traditional Mediterranean diet: contribution of traditional dishes to the Lebanese diet, Proceedings of the Nutrition Society, Vol. 67, E170, 2008.

[16] M. Tueni, A. Mounayar, and I. Birlouez-Aragon, Development and evaluation of a photographic atlas as a tool for dietary assessment studies in Middle East cultures, Public Health Nutrition, Vol.15, No.6, 1023-1028, 2012.

[17] The National Survey of Household Living Conditions (Lebanese Republic Ministry of Social Affairs, Central Administration for Statistics and UNDP), 2004.

[18] P.L. Pellet, S. Shadarevian, Food Composition Tables for Use in the Middle East, 2nd ed. Beirut: Division of Food Technology and Nutrition, Faculty of Agricultural Sciences, American university of Beirut, Lebanon, pp. 116, 1970 (English).

[19] U.S.D.A., National Nutrient Database for Standard Reference, Release 18 2005, Online available from, http://www.nal.usda.gov/fnic/foodcomp. 
[20] J.D. Fernandez-Ballart, J.L. Pinol, I. Zazpe, D. Corella, P. Carrasco, E. Toledo, M. Perez-Bauer, M.A. Martinez-Gonzalez, J. Salas-Salvado, J.M. Martin-Moreno, Relative validity of a Semi-Quantitative Food-Frequency Questionnaire in an elderly Mediterranean population of Spain, British Journal of Nutrition, Vol.103, 1808-1816, 2010.

[21] F. H. Esfahani, G. Asghari, P. Mirmiran, F. Azizi, Reproducibility and Relative Validity of Food Group Intake in a Food Frequency Questionnaire Developed for the Tehran Lipid and Glucose Study, Journal of Epidemiology, Vol. 20, No.2, 150-158, 2010.

[22] V. Deschamps, B. de Lauzon-Guillain, L. Lafay, J.M Borys, M.A Charles, M. Romon, Reproducibility and relative validity of a food-frequency questionnaire among French adults and adolescents, European Journal of Clinical Nutrition, Vol. 63, 282-291, 2009.

[23] A. Affret, D. El Fatouhi, C. Dow, E. Correia, M.C. Boutron-Ruault, G. Fagherazzi, Relative Validity and Reproducibility of a New 44-Item Diet and Food Frequency Questionnaire Among Adults: Online Assessment, Journal of Medical Internet Research, Vol.20, No.7, e227, 2018.

[24] E. O. Verger, P. Armstrong, T. Nielsen, R. Chakaroun, J. Aron-Wisnewsky, R. J. Gøbel, T. Schütz, F. Delaere, N. Gausseres, K. Clément, B.A. Holmes, on behalf of the MetaCardis Consortium, Dietary Assessment in the MetaCardis Study: Development and Relative Validity of an Online Food Frequency Questionnaire, Journal of the Academy of Nutrition and Dietetics, Vol.117, 878-888, 2017.

[25] M.T. Streppel, J. De Vries, S. Meijboom, M. Beekman, A. De Craen, P.E. Slagboom, E. Feskens, Relative validity of the food frequency questionnaire used to assess dietary intake in the Leiden Longevity Study, Nutrition Journal, Vol.12, 75, 2013.

[26] V. Bountziouka, E. Bathrellou, A. Giotopoulou, C. Katsagoni, M. Bonou, N. Vallianou, J. Barbetseas, P.C. Avgerinos, D.B. Panagiotakos, Development, repeatability and validity regarding energy and macronutrient intake of a semi-quantitative food frequency questionnaire: Methodological considerations, Nutrition, Metabolism and Cardiovascular Diseases, Vol.22, No.8, 659-667, 2012.

[27] M.J. Lombard, N.P. Steyn, K.E. Charlton, M. Senekal, Application and interpretation of multiple statistical tests to evaluate validity of dietary intake assessment methods, Nutrition Journal, Vol.14, 40, 2015.

[28] J. Vioque, E.M. Navarrete-Muñoz, D. Gimenez-Monzó, M. García-de-la-Hera, F. Granado, I. S. Young, R. Ramón, F.
Ballester, M. Murcia, M. Rebagliato, C. Iñiguez, Reproducibility and validity of a food frequency questionnaire among pregnant women in a Mediterranean area, Nutrition Journal, Vol.12, 26, 2013.

[29] W. De Keyzer, A. Dekkers, V.V Vlaslaer, C. Ottevaere, H.V. Oyen, S. De Henauw, I. Huybrechts, Relative validity of a short qualitative food frequency questionnaire for use in food consumption surveys, European Journal of Public Health, Vol. 23, No.5, 737-742, 2012.

[30] N. Mohammadifard, F. Sajjadi, M. Maghroun, H. Alikhasi, F. Nilforoushzadeh, N. Sarrafzadegan, Validation of a simplified food frequency questionnaire for the assessment of dietary habits in Iranian adults: Isfahan Healthy Heart Program, Iran, ARYA Atherosclerosis, Vol. 11, No. 2, 139-146, 2015.

[31] E. Athanasiadou, C. Kyrkou, M. Fotiou, F. T. Sakoumaki, A. Dimitropoulou, E. Polychroniadou, G. Menexes, A.P. Athanasiadis, C.G. Biliaderis, A.M. Michaelidou, Development and Validation of a Mediterranean Oriented Culture-Specific Semi-Quantitative Food Frequency Questionnaire, Nutrients, Vol.8, No.9, 522, 2016.

[32] R.F. Tayyem, S.S. Abu-Mweis, H.A. Bawadi, L. Agraib, K. Bani-Hani, Validation of a food frequency questionnaire to assess macronutrient and micronutrient intake among Jordanians, Journal of the Academy of Nutrition and Dietetics, Vol.114, No.7, 1046-1052, 2014.

[33] K. El Kinany, V. Garcia-Larsen, M. Khalis, M. Mint Sidi Deoula, A. Benslimane, A. Ibrahim, M.C. Benjelloun and K. El Rhazi, Adaptation and validation of a food frequency questionnaire (FFQ) to assess dietary intake in Moroccan adults, Nutrition Journal, Vol.17, No. 61, 2018.

[34] L. Natarajan, M. Pu, J. Fan, R. A. Levine, R. E. Patterson, C. A. Thomson, Pierce, J. P, Measurement Error of Dietary Self-Report in Intervention Trials, American Journal of Epidemiology, Vol.172, No.7, 819-827, 2010.

[35] S. Buscemi, G. Rosafio, S. Vasto, F. Maria Massenti, G. Grosso, F. Galvano, N. Rini, A.M. Barile, V. Maniaci, L. Cosentino, and S. Verga, Validation of a food frequency questionnaire for use in Italian adults living in Sicily, International Journal of Food Sciences and Nutrition, Vol.66, No.4, 426-438, 2015.

[36] J. Cantin, E. Latour, R. Ferland-Verry, S. Morales Salgado, J. Lambert, M. Faraj, A. Nigam, Validity and reproductibility of a food frequency questionnaire focused on the Mediterranean diet for the Quebec population, Nutrition, Metabolism and Cardiovascular Diseases, doi: 10.1016/j.numecd.2015.11.003, 2015. 